\title{
Intelligent computer-assisted instruction
}

\author{
RICHARD MILLWARD, LOU MAZZUCCHELLI, SCOTT MAGOON, \\ and REBECCA MOORE \\ Brown University, Providence, Rhode Island 0291I
}

\begin{abstract}
A description is provided for an intelligent computer-assisted instruction system (BANDAID) which is in an early stage of development.
\end{abstract}

The title of this paper is rather presumptious for at least two reasons. First, we are just beginning to build an "intelligent" computer-assisted instruction system (BANDAID) and have reached a preliminary stage in which we have a good interactive parser, editor, and interpreter for BASIC. The source of the intelligence for this program is the second reason I say we are rather arrogant. Intelligence for a CAI system is not a standard commodity that one orders from some catalog. Some ideas exist for what we might mean by "intelligence" and it is those ideas, and a few of our own, that constitute the intelligence in our system. Another possible source of "intelligence" for a CAI system is dialogue analyses of interactions between a tutor and his tutee. We plan to make such analyses a standard part of the development of our CAI system and thus build our intelligence up from data as well as down from ideas. Most of the ideas for this system have already been tried; some of them we borrowed.

There are two important references for our work. One concerns the use of tutorial dialogues for analysis of the tutor-tutee interaction. Collins, Warnock, and Passafiume (1975) describe a number of strategies used by tutors in a teaching session on geography in South America using the SCHOLAR system. Since we have space to discuss only our system, we will not review the results of that seminal paper, but we strongly urge the reader to do so. The central idea here is to place a human tutor into the CAI system to provide it with intelligence and then to analyze the sessions to formulate a theory of a tutor's role in CAI. We will gradually transfer the human tutor's role to the CAI system.

The second important source of information comes from the work of Brown and Burton (1975). Their CAI system, SOPHIE, differs from the traditional one in that it is not used just to "teach," that is, to present material to a student; it is also used as an expert to which the student can turn for information and suggestions. A SOPHIE session is conducted in fairly natural English. The basic task for a student interacting with SOPHIE is to find a bug in an electronic circuit. The student can ask for information about the circuit and suggest the source of the error to the program. The program monitors the information the student has received and determines whether he has tested enough components to determine the source of the bug in the circuit. SOPHIE has inferencing capabilities, hypothesis-generation procedures, and decision-making components that make it the most "intelligent" CAI system around today. An important innovation in SOPHIE is the idea of a dialogue between the student and the TUTOR rather than having the TUTOR simply present problems or facts to which the student passively responds. We plan to incorporate this innovation into our system. The hypothesis-generation and inferencing capabilities are also necessary components of a truly interactive system, and we intend to put some of these components into BANDAID. However, just because these things have been done does not mean they are easy. On the other hand, because they have been done, our sights should be aimed at least as high as the present state of the art.

One final comment before specifying the details of the BANDAID system. Although writing an intelligent CAI system to teach BASIC is applied, it is the kind of applied work that needs no apology by even the most theoretically oriented psychologist or the most empirical experimentalist. First, putting together a complex system that understands even simple English, that models the knowledge of a student in a subject matter as complicated as a programming language, and that guides a student through simple exercises by correcting his misinterpretations of code and program goals is no mean task. It is also a very decent theoretical exercise since many of the current problems in cognitive psychology must be solved in order to implement an intelligent CAI system. Armchair philosophizing about these problems is not sufficient because the system must work. Finally, as one tries to build such a system, the questions that arise require a great deal of empirical research.

\section{BANDAID}

BANDAID is a contrived acronym. BANDAID will run on the IBM 360 computer under MTS LISP, provided by Carole Hafner and Bruce Wilcox of the Mental Health Research Institute at the University of Michigan. It is a system with a number of components, some of which are implemented and some of which are not. The parts of the system that are theoretical will eventually be implemented in stages. In this paper, we will 


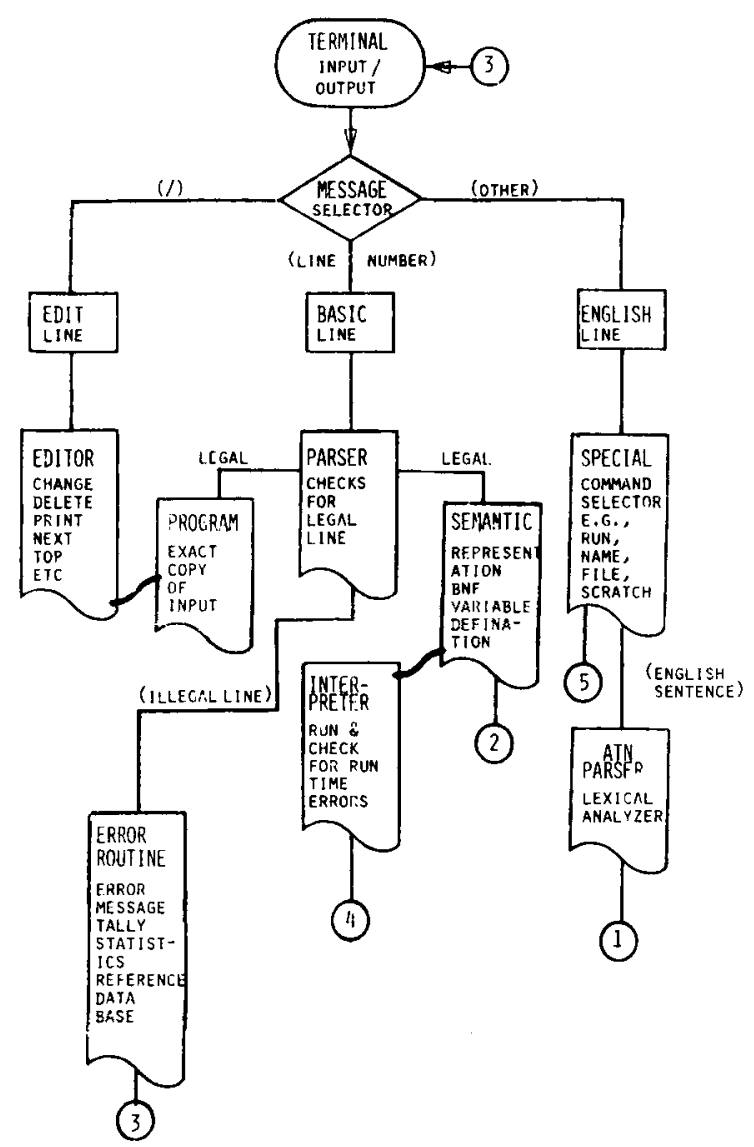

Figure 1. The BASIC language components of BANDAID.

spend a fair amount of time on what is implemented and leave the rest for later talks. However, we do feel that an overview of the total system and our plans for research using it should at least be mentioned at this time.

\section{The BASIC Language System}

Figure 1 is a diagram of the portion of the system that is almost completed. It is a very sophisticated programming system for novice and expert programmers. It is not a programming system on which to make production runs because the whole system is simulated in LISP. How this is done will be discussed later. The major components are the message selector, the parser, semanttic representation, the interpreter, the editor, and the ATN English parser.

A student sits at a terminal and enters one of three types of lines: BASIC lines, which always begin with a number; edit lines, which begin with a slash; and English lines, which are parsed by the ATN parser. Selecting the control commands of BASIC, RUN, SCRATCH, etc., is no problem since they are simply keywords. The program that the student inputs is analyzed as follows: First, the BASIC line box extracts the line number from the input line and creates an atom of it. Since numbers enter as ASCII characters, this presents no problem. Then the statement following the line number is set as the value of the line number. All blanks are removed from this line and the remaining string is sent to the parser, which checks the line for syntax. Errors lead to error messages and, when eventually implemented, to corrective comments, statistics about the student, etc. A correct line is then converted into a semantic representation. This semantic representation is placed on the P-LIST of the line-number atom. It contains two indicators, COMMAND and PARMS. The interpreter takes the P-LIST of each atom and performs the command as defined in the parameter list. The interpreter has two modes, CHECK and RUN. The CHECK mode looks for semantic errors such as undefined variables or functions, no dimension statements, etc. The RUN mode runs the program.

This is obviously not a production system, since every command will be run by LISP in an interpretive mode. What are the arguments for doing it this way? First, we want a system that is easily modified. Therefore, our data structures and the interactions of various modules should be clearly defined as functionally distinct units. When the system is running the various components of the system will interact in arbitrary ways and we cannot preset these interactions without severely restricting the system. Also, the various components must interact easily with one another. For example, because we are teaching students BASIC on an IBM machine and on a DEC machine, one idea is to develop a superset of instructions derived from the two somewhat incompatible systems. IBM has matrix operations and DEC does not; DEC has excellent string-handling functions and IBM none. With the LISP implementation of the interpreter, a student simply types in IBM and then he is allowed to use only the IBM subset of instructions. Future expansions of the language can be added easily but restricted in their use in the same way. Thus, novice programmers can be limited to simple BASIC and advanced programmers can be given BASIC PLUS by simply typing BASIC PLUS.

The ordinary way to treat a student program with a compiler provides little information about the program and about run-time bugs. We want to be able to "look" at the program as it is being run. This means tracing instructions and variables, making snapshots, selecting certain instances through a loop, halting at certain lines, etc. This can be done easily with an interpreter in a higher level language such as LISP. We can tag each linenumber atom, indicating whether that line should be listed as it is run, listed every 10th time it is run, omitted in a run, substituted for by a temporary line, etc. Likewise, variables can be tagged so that as they are changed, the line number being run and the old and new values are printed out.

A third important advantage of interpreting the student's BASIC code has to do with the more advanced features of BANDAID. Eventually, we want to make it possible for a student to ask about his own program. For example, "Have I used A anywhere?" "Give me a 
list of the subscripted variables I have used." "What does a flowchart of my program look like at this point?" To do this, we will have to search through the semantic representation of the program on the P-LIST of each line number to get the kind of information requested.

Finally, an instructor grading a student program does not simply look to see if it conforms to some fixed pattern, but rather analyzes it to see if it does the job correctly and whether it is efficient. Such evaluation is not easy because it requires abstracting the essence of the program. This requires creating a representation of a program that is more abstract than the specific lines of codes and their order. The system has to understand equivalent representations of a solution to a programming problem. Looking at a program in this way requires more than a compiler. These changes can be put in temporarily under the user's control without elaborate special instructions to indicate they are there.

Finally, there will be a number of pieces of the program-the ATN parser, for example-that will be general and not specific to the BANDAID system. We want these units to be usable in other programs in a general way. Using LISP helps to insure this kind of flexibility.

\section{The TUTOR System}

Figure 2 presents a schematic diagram of the future parts of the system. We cannot indicate specifically how we will implement each of these components because we are not yet sure ourselves. Each box represents a serious challenge in design, and the integration of the various boxes will also present a number of problems. However, some idea of the overall structure is necessary now to insure that, as each model is constructed, its interface to other models is not ignored.

The semantic interpreter will accept the output of the ATN parser and build a semantic structure that can be interpreted by the control system. At first, of course, only rather elementery English sentences will be accept- ed, but as the system develops, more general English language forms will be understood. We need English input to allow questions about the program itself as well as about BASIC. A knowledge network will encode facts about the BASIC language. These facts will consist of syntactic rules, semantic facts about BASIC statements, and more general pragmatic facts such as rules for loops (nesting must be embedded, FOR-TO index variables cannot be subscripted), when to use a function definition rather than a subroutine, how to program a simple sort routine, and how to extract individual digits from a number. Representing such knowledge so that it can be retrieved by English commands is not easy. In the past, many CAI programs had canned paragraphs of text that were spewed out to the student on the basis of some keyword match. SOPHIE shows that we can be more subtle than that. But the less constrained the knowledge base, the more difficult it is to answer questions, and BASIC appears more complex than simple fact retrieval. Consider a possible statement by a student user: "I don't understand printing variables." This has to be interpreted as "I don't understand how to print variables," since there are no "printing variables." Then there is the problem of answering the question (which was not stated as a question) since it is not clear exactly what is not understood. Presumably, context is important here; if an input line " 120 PRINT A B C" was just returned with an error message, then the answer would concern delimiters between variables in a PRINT statement. Eventually, we plan to tie the knowledge base network and the error routine together so that an error will generate a meaningful comment about the reason for the error.

The expanded system will have a set of exercises to present to students. Here we can do no better than follow Wescourt, Beard, and Bould's (in press) knowledge-based CAI system at Stanford. Their system allows for a very flexible selection of problems that depends on how fast the student progresses. It contains

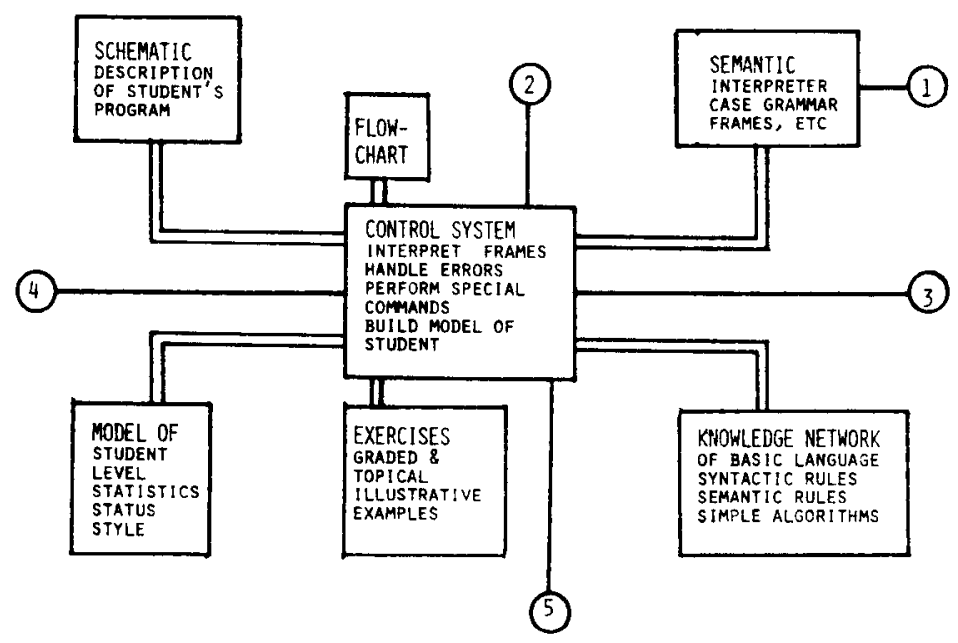

Figure 2. The TUTOR components of BANDAID. 
a model of the student in the sense that it has a set of statistics for that student. We hope to produce a more elaborate and more psychologically realistic model of the student. To do this, we will have to understand the somewhat vague concept of "style." To give one simple example which is fairly easy to handle, consider the question of the kind of problems to give a student. If the student has a background of calculus and physics, one can use as examples fairly complicated mathematical problems. On the other hand, if he is a business student, the examples should involve less pure mathematics. Selecting the right set of problems for a student is not a trivial task; often a student has trouble solving a problem only because he does not know the algorithm necessary for solving it. Of course, one aspect of programming-the hardest, in fact-is developing algorithms for problems. But that should not be the stumbling block for a beginning student, although it often is.

The final component of the system pictured in Figure 2 is the schematic description of the BASIC program. Our plans are to build a structure that transcends the particular variables used, the order of computing subparts of the program, and the various ways of implementing certain basic algorithms. When a grader looks at a student's program, his evaluation is based not on whether it matches some prototype program, but on whether it does what it is supposed to do. Then, there are inefficiencies in the implementation to be considered. How can a program be represented so that a student's program can be compared to it? This is no simple question and a number of research groups are working on such problems under the rubric of automatic programming. Again, we ask you to consider a box like the schematic description not as a "functioning part" to be built on demand, but rather as an idea component of the total system. Our implementation will start by producing a simple flowchart of the program. That much seems relatively easy. Then we will try to build more abstract characterizations of the program.

\section{TUTORIAL DIALOGUES}

The BASIC language system of BANDAID will be used to conduct some experiments designed to learn more about tutorial situations. We all believe that instruction is generally better if a tutor works directly with a student. There are a number of obvious explanations why this is probably true and most make good sense: The student can ask questions when he is confused, his mistakes are given immediate feedback, and the material is presented at a pace appropriate to the student's abilities. But these are rather general ideas about the tutorial situation, and a science of instruction needs to have a more explicit body of knowledge about tutorial instruction in order to apply tutorial "intelligence" to CAI systems.

The purpose of studying tutorial dialogues is, first, to learn more about the role of the tutor in teaching and, second, to develop a model of the tutor that can be placed into the BANDAID system. This will be done by having the "intelligent" part of BANDAID be a live tutor who takes over from the computer when it is not capable of answering a student's questions. Step by step we will try to shift the functions of the live tutor into the BANDAID system. An important aspect of this arrangement is that the system will have "intelligence" right from the beginning, even though we cannot implement such intelligence into a CAI system itself. At the same time, we can learn more about what we mean by "intelligence" while gathering data about the tutorial situation.

Figure 3 presents the design of the "tutorial" laboratory. The human tutor and his tutee sit in the experimental room in booths about $5 \mathrm{ft}$ apart. Each has a teletypewriter keyboard and a TV monitor with 16 lines. Optionally, each wears earphones and has a microphone connected so that the tutor can talk to the tutee through the latter's earphones, and vice versa. Both conversations are recorded on independent channels of a stereo tape recorder. We say "optionally" because some experiments will not use the voice communication and others will. Since there will be no spoken interaction in the final system, we want to phase it out as soon as possible. On the other hand, we do not want to overly constrict the tutor-tutee interaction until we learn more about its structure (cf. Chapanis, 1975).

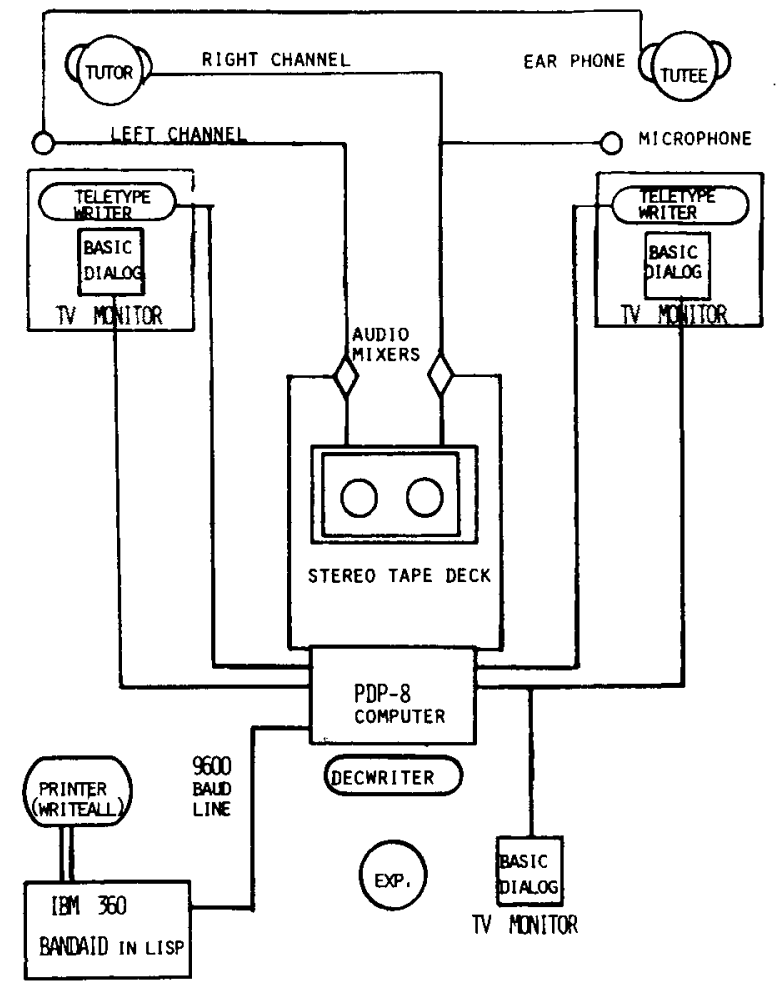

Figure 3. The computer system to be used for tutorial dialogues. 
The TV monitors are connected to a PDP-8 computer with the usual DECwriter for the experimenter to use. He also views the TV monitor and, optionally, can listen in on the two conversations. The PDP- 8 computer is connected to the IBM 360 on which LISP is running BANDAID.

An important software component of this system is WRITEALL. Our IBM system has a monitor command that will take all lines printed on a terminal and write them into a file which then can be off-line printed. We will create a software system on our PDP.8 which is similar to WRITEALL but with some important new features. First, we will write the time out every $n \mathrm{sec}$ (usually $\mathrm{n}$ will be 60 ). Second, the typed dialogue between the tutor and the tutee will be written on the file along with the interactions between the student and BANDAID. Finally, the spoken communications recorded on the two channels of the tape recorder will be overlaid with a beep at every time unit. A special program to be run after the session is over will allow the experimenter to take the spoken recording and type in the dialogue so that it merges with the previously recorded typed interactions between the tutor and tutee. This can be easily done because of the time marks placed on both the spoken and the typed records. The final file containing BANDAID lines, typed dialogue lines, and typed-in spoken lines will be sent to the IBM 360 at a 9,600-baud rate, where it will be written to tape and also printed on the high-speed printer.

These records will then serve as data to be studied for ideas about how a tutor teaches. Simple statistics about the number and types of queries made by students, about the amount of information provided by a tutor, about the strategies used by the tutor, the organization of information implied by the tutor's presentation, etc., will be gleaned from these elaborate protocols.

In some pilot experiments with this system, we have discovered that it is a very pleasant and effective way to teach. Students like the fact that they have instant answers to their questions and at the same time can be interacting with their program. The mechanisms of running a BASIC program (the control commands and file manipulation problems) are easily handled. It is very hard to tell a student about CLEAR and EXIT on a blackboard in front of a class but very natural and easy to do in an interactive dialogue situation on a terminal.

In summary, then, it is the information gained from these tutorial sessions that will form the basis of new components to BANDAID. As mentioned earlier, some of these components are very ambitious in scope but rather hazy at this point. We will use the tutorial dialogue sessions to learn about what students expect from such a system, how tutors handle the standard questions raised, and the kind of model the tutor forms of his student and how that model helps him teach that student. The details of our experiments are not presented here since they constitute a different aspect of this research, which I hope to report on next year.

Some time ago, Licklider mentioned that the computer will not so easily take over man's intelligent functions as it will become an extension of his intelligence by providing skills in which man himself is limited. The tutorial dialogue scheme is a propadeutic example of this symbiosis.

\section{REFERENCES}

Brown, J. S., \& Burton, R. R. Multiple representations of knowledge for tutorial reasoning. In D. G. Bobrow \& A. Collins (Eds.), Representation and understanding studies in cognitive science. New York: Academic Press, 1975.

Chapanis, A. Interactive human communication. Scientific American. 1975, 232. 36-42.

Collins, A., Warnock, E. H., \& Passafiume, J. J. Analysis and synthesis of tutorial dialogues. In G. H. Bower (Ed.), The psychology of learning and motivation (Vol. 9). New York: Academic Press. 1975.

Wescourt, K. T., Beard, M., \& Gould, L. Knowledgebased adaptive curriculum sequencing for CAI: Application of a network representation. Proceedings of the National Association of Computing Machinery, in press. 